\title{
Juegos de escape en educación
}

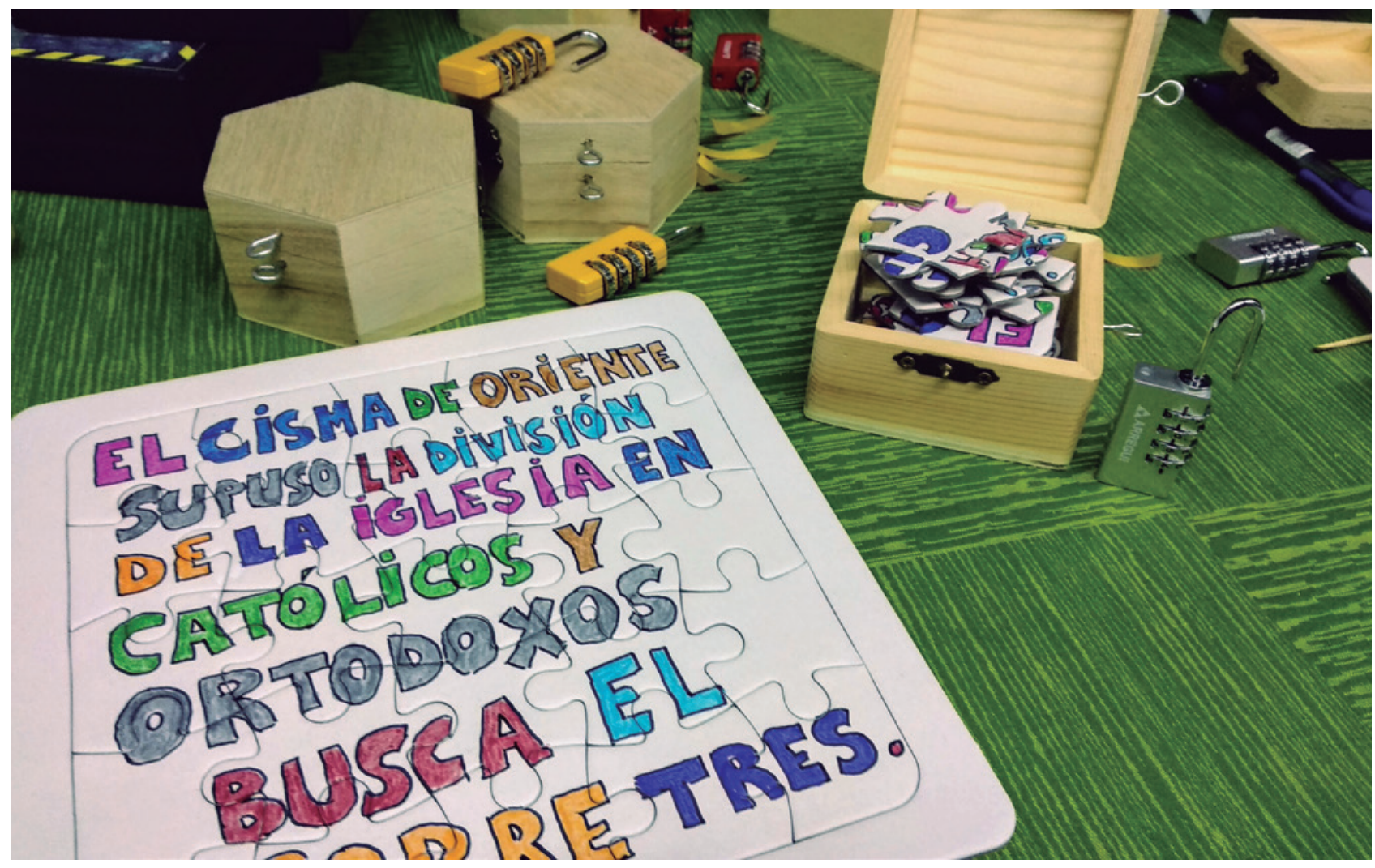

Hace unos años la fiebre por los juegos de escape irrumpió como una actividad social lúdica y es ahora cuando se está trasladando al ámbito educativo con bastante aceptación entre alumnos y profesores. En el presente artículo se dan algunas pinceladas para conocer con mayor profundidad en qué

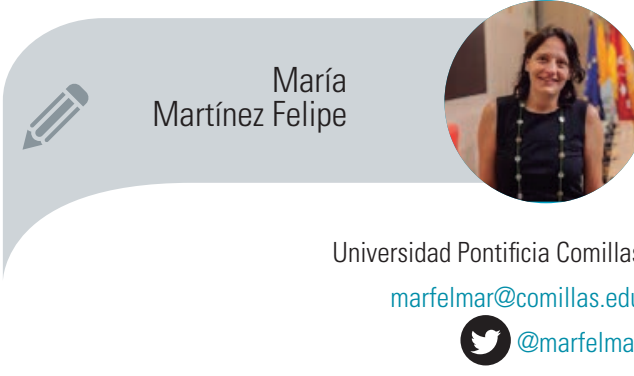
consiste y algunas orientaciones didácticas

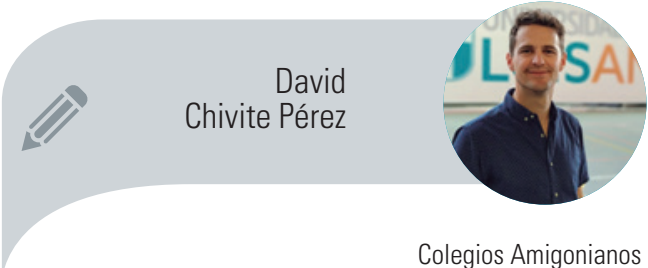
Colegios Amigonianos
dchivite@gmail.com (y) @dchivite para poder implementarlo en el aula. 


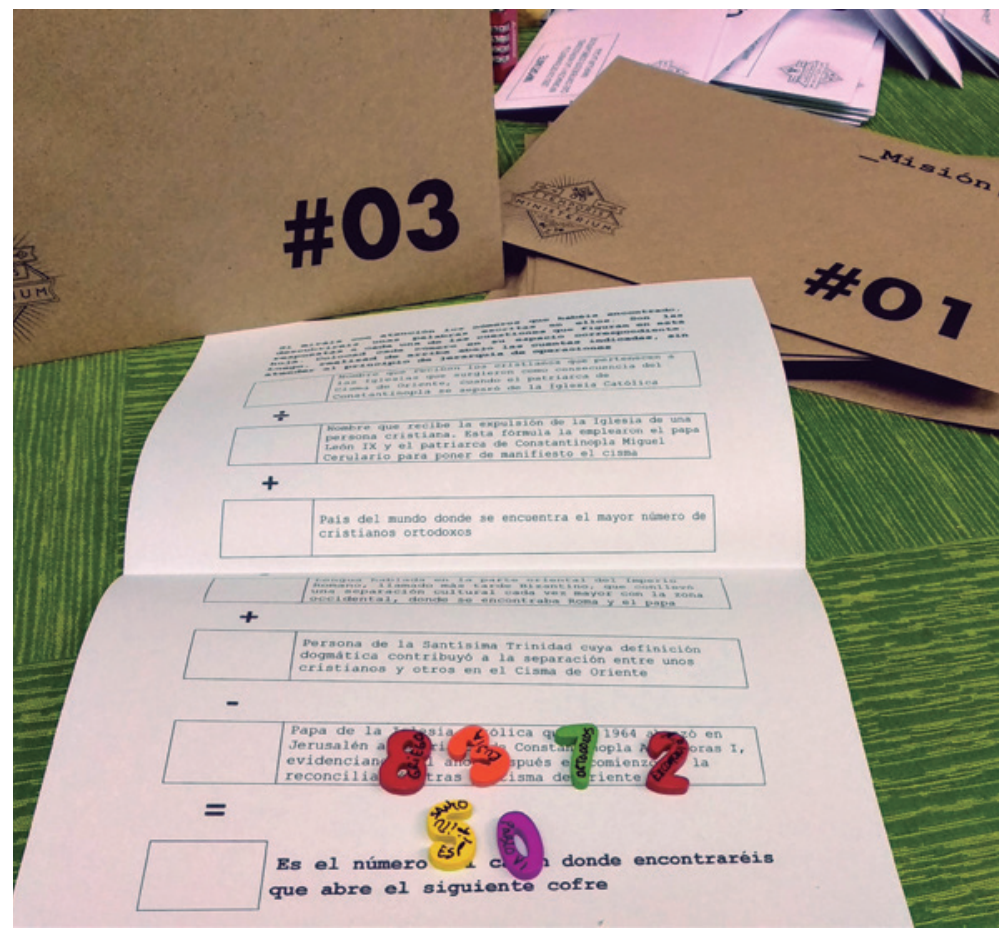

¿Qué es un juego de escape?

Bajo la denominación castellana de juegos de escape se encuentran asociados dos términos en inglés: Breakout EDU y escape room. Ambos términos hacen referencia a un conjunto de herramientas de gamificación donde un grupo de personas, normalmente agrupadas en equipos, compiten para resolver una serie de retos con el fin de salir de un espacio concreto en un tiempo determinado. En algunos contextos se han considerado a ambos términos como sinónimos, pero, hay cierto matiz que los diferencia como bien apunta José Luis Redondo', docente de Ciencias Sociales, en su blog personal.

El matiz diferenciador es que escape room (habitación de escape) surgió de un modo ajeno al ámbito educativo y consiste en que un grupo muy reducido de personas se encierra en una serie de estancias y se puede contar con una interlocución exterior, ya sea a través de una cámara, ordenador, teléfono o altavoz, para orientar a los participantes si fuese necesario.

En cambio, un Breakout EDU no se limita a salir de una estancia en concreto, sino que el reto consiste en abrir diferentes cajas cerradas con diferentes tipos

1 Redondo, J. L. (2017, 16 de diciembre). ¿BreakoutEDU o Escape Room? \#Gamificación analógica y educativa. [Mensaje en un blog]. José Luis Redondo. Recuperado de https://bit.ly/2XLQypu de candados a través de la resolución de problemas, cuestionarios y enigmas que están contextualizados en una narrativa concreta. A nivel educativo tiene una ventaja primordial y es que permite multiplicar el mismo reto varias veces creando diferentes desafíos que conduzcan al mismo fin. Esto nos permite que toda una clase, dividida en varios grupos reducidos pueda resolver un mismo Breakout EDU sin necesidad de tener una única estancia por grupo o de tener esperando al resto de alumnos mientras un grupo ocupa dicha estancia donde están todos los retos.

Aunque en esencia estas son las características que definen los juegos de escape, a la hora de ponerlo en práctica, nos podemos encontrar diferentes variantes: versión analógica o digital, versión individual o grupal, etc.

Este tipo de estrategias lúdicas inmersivas han tenido una gran acogida en el ámbito educativo ya que favorecen el desarrollo de diversos aspectos pedagógicos tal y como se presenta a continuación.

\section{Potencialidades educativas}

Al participar en este tipo de juegos, el alumno gestiona su propio aprendizaje y, a su vez, permite el desarrollo de los siguientes aspectos:

入 Desarrollo cognitivo: lógica, memoria, atención, concentración, creatividad, agilidad mental, resolución de problemas, gestión del tiempo y de los recursos disponibles, entre otros.

入 Desarrollo de habilidades sociales: trabajo en equipo, cooperación, asertividad, coordinación, liderazgo, entre otros.

त Desarrollo afectivo: expresión y control de emociones, refuerzo del sentimiento de logro, sentimiento de pertenencia a un grupo, refuerzo de la autoestima, entre otros.

入 Desarrollo educativo: motivación, mejoras en el aprendizaje, asentamiento de aprendizajes previos, así como el desarrollo de diversas competencias como la comunicación lingüística, aprender a aprender, competencias sociales y 
cívicas y el sentido de la iniciativa y espíritu emprendedor o las competencias propias de su diseño (área matemática, lingüística, social...), entre otros.

7 Desarrollo psicomotor: coordinación psicomotriz (fina y gruesa), flexibilidad, fuerza, resistencia, entre otros.

Una vez presentadas algunas de las potencialidades educativas, se procede a definir su estructura y los pasos a seguir para aplicarlo en el aula.

\section{Consideraciones previas}

Antes de diseñar el juego de escape, sería interesante que se formará un equipo y se experimentara en vivo en qué consiste o, al menos, familiarizarse con este tipo de juegos. Con el fin de que sea una experiencia didáctica lo más enriquecedora posible, se han de tener en cuenta las siguientes recomendaciones, tal y como se recoge en el Manual de Diseño de un Juego de Escape elaborado por la Junta de Extremadura (2018): y Genera una trama que permita motivar a los alumnos y meterles en situación. Para ello es esencial que la narrativa y la complejidad de los retos se adapten a sus capacidades e intereses. La implicación de los alumnos dependerá en gran medida de la consistencia y la motivación que genere la narrativa y que deberá estar acompañada de una estética atractiva. Los conceptos o narrativas más frecuentes según Nicholson $(2015)^{2}$ son los siguientes: Escapar de algún lugar o liberar a alguien; Cometer un atraco; Misión de espionaje; Investigar un crimen o misterio; Desactivar un artefacto explosivo; Encontrar a alguien desaparecido; Ayudar a crear algo (una poción, un antídoto, una cura...); Evento sobrenatural; Operaciones militares. Si lo quieres desarrollar dentro del ámbito de las Ciencias Sociales, puedes contextualizarlo en una época o acontecimiento histórico o trabajar algún contenido de Geografía expuesto por algún explorador o científico.

y Crea un personaje que contextualice de una forma épica la trama que se desarrollará y que presente instrucciones y plantee un reto al equipo.

\ Identifica los objetivos didácticos, los componentes que tendrán que utilizar para resolver el reto y el número de retos acorde con el tiempo de la sesión. Se recomienda que la sesión no supere los 45 minutos de duración y que contenga de cinco a siete retos diferentes.

y Diseña, prueba y rediseña los retos. Parte de una idea que te ayude a ir dando forma a tu historia y puedas mejorar su diseño con ideas sucesivas. Los retos pueden ser independientes unos de otros (lo que simplifica el diseño) 0 estar ligados entre sí con la propia narrativa, lo cual hace que sea más motivador ya que la narrativa proporciona un feedback automático cada vez que se supera un reto. Es importante que los retos sean diferentes para que no se copien entre sí y que la dificultad sea equitativa entre los grupos y acordes con su edad.

У Sé concreto. Empieza por algo sencillo, adaptado al espacio y los materiales disponibles.

Establece reglas claras (el tiempo del que disponen, el objetivo final, cómo solicitar pistas, etc.) y, si es necesario, acompaña cada reto de carteles indicativos o pistas para su comprensión. No des nada por obvio.

Nicholson, S. (2015). Peeking behind the locked door: A survey of escape room facilities. Disponible en: http://scottnicholson.com/pubs/erfacwhite.pdf

Después de este rápido repaso por las cuestiones previas, centremos nuestra mirada en cómo diseñarlo.

\section{Cómo diseñar un juego de escape}

A la hora de diseñar un juego de escape educativo es necesario tener en cuenta qué se ha de hacer antes de entrar en la estancia, durante el desarrollo y al terminar la actividad.

\section{Antes de entrar en la estancia}

त Es importante conocer el espacio y el número de participantes. Distribuye a tus alumnos en grupos que oscilen entre tres y ocho participantes.

7 Ofrece información general centrada en el uso de la sala, su contenido, reglas del juego, tiempo disponible y cómo solicitar pistas. Además, se avisa que, si alguien se siente indispuesto, puede abandonar la sala y dar por terminada la actividad.

7 Se recomienda mostrar el tipo de candados que encontrarán y hacer una demostración de su apertura.

入 Se pueden dar algunas indicaciones que mejoren el desarrollo de la actividad como, por ejemplo, que la comuni- 
У Aunque el artículo está enfocado para ser desarrollado en el aula con los alumnos, también puede ser una buena oportunidad para desarrollarlo con el equipo directivo 0 con el profesorado para afianzar las relaciones personales y profesionales, mejorar el trabajo en equipo por etapas, ciclos 0 áreas, así como fortalecer el sentimiento de pertenencia a un grupo.

$\checkmark$ Varios profesores comparten con gran generosidad sus materiales, su experiencia, y sus recursos en sus blogs o páginas web personales. Aquí van algunos enlaces interesantes:

I [Applejux]: lista abierta de materiales básicos para crear un kit Breakout y recopilación de experiencias de docentes en español, gallego, euskera 0 catalán disponibles en la red recopilados por el profesor cHristian Negre: https://www.applejux.org/teacher/

I [Ágora Abierta]: web creada por la profesora Clara Cordero que recopila materiales y orientaciones para su aplicación didáctica en el aula: https:// www.agorabierta.com/2018/03/escape-room-educativo/

I [Proyecto Looking @ Learning]: repositorio de juegos de escape educativos: https://lookingatlearning,eu/eduescperooms/

Existen varios juegos de escape gratuitos para disfrutar en familia desde casa recopilados por la web de Educación 3.0. Cada miembro de la familia puede convertirse en un integrante de la escuela de magia de Harry Potter o ponerse en la piel de un científico que está investigando la vacuna para acabar con el coronavirus: https:// www.educaciontrespuntocero.com/ocio/escape-rooms-gratuitos/

cación entre todos facilita el trabajo grupal, que la información se debe compartir entre los miembros del grupo o que los objetos utilizados son de un único uso (si una llave u objeto se ha utilizado para un enigma, no servirá para otro diferente), por lo que se recomienda ponerlos a parte del resto de objetos.

入 Ofrece información relacionada con la trama del juego, sobre lo que van a encontrar cuando entren en la sala. Se recomienda ambientar la estancia acorde con la temática y caracterizar al personaje. En algunos casos, se utiliza un vídeo introductorio a modo de mensaje o tráiler.

入 Se puede entregar algún tipo de material personalizado (contrato de exención de responsabilidades, carta con algún dato, carné, etc.) que estén ambientados con la estética de la trama.

\section{Durante el desarrollo}

Los alumnos tendrán que explorar la estancia para descubrir cualquier in- formación en forma de objetos, pistas o puzles, que permita resolver los retos que les guíen hacia el objetivo final. Para ello tendrán que ayudarse del trabajo en equipo, la comunicación, la lógica, el razonamiento deductivo y analítico, o la destreza psicomotora, entre otros. A continuación, señalaremos algunos de los más frecuentes:

a) Componentes: entre los objetos que configuran los componentes de cada reto, destacamos los siguientes: candados, cajas de seguridad, cartas de juego, códigos, códigos QR, cofres, cuadros, dibujos, dispositivos electrónicos, escondites (latas de refrescos, libros huecos, piedras falsas, dobles fondos...), espejos, experimentos, fotos, libros, luz negra, llaves, maletines, memorias USB, pósteres, prismáticos, relojes, rompecabezas, tinta invisible, etc.

b) Pistas: al estar dentro de la estancia, los alumnos estarán aislados y en el caso de que se bloqueen, se puede preparar un sistema de ayuda para cada uno de los retos. Se establece previamente cómo será dicho sistema (si se puede limitar el número de pistas, esperar un tiempo determinado para solicitarlo, si tiene o no repercusión en el juego, etc.). Las pistas pueden ser solicitadas a través de botones o campanas, mediante voz, o a través de un sobre.

c) Tipos de puzle: los tipos de puzles que se pueden encontrar en la habitación pueden ser de distintos tipos tal y como se muestra en la tabla 1.

\section{Después de terminar la actividad}

El juego de escape se da por finalizado cuando el grupo ha logrado el reto o cuando ha transcurrido el tiempo establecido. En el momento en que los participantes consiguen gestionar bien su tiempo y hacen un uso eficaz del arsenal de habilidades personales y sociales y lo ponen al servicio del aprendizaje, es cuando neutralizan el desafío planteado. Es, en este punto, cuando este tipo de 
Tabla 1. Tipos de puzle

TIPO DE PUZLE

Búsqueda de objetos escondidos en la habitación

Resolver una situación con ayuda de otros

Luz

Recuentos

Detectar información expuesta

Información en un texto donde se resalten letras o palabras

Sustitución de símbolos con una clave

Códigos sin una clave

Empleo inusual de algún objeto

Ensamblaje de objetos físicos (rompecabezas)

Identificación de patrones

Acertijos

Audio

Espejos

Coordinación ojo-mano

Lógica abstracta

Búsqueda de información usando fuentes

Cuerdas o cadenas

Crucigramas tradicionales

Recuperar objetos fuera de nuestro alcance

Laberintos

Palpamiento

Manipulación de formas

Interacción con actores

\section{EJEMPLO}

Se deja una carta, un sobre, un mensaje en una pizarra... que lleva a resolver un puzle o desbloquear un candado

Un jugador ejerce fuerza sobre una trampilla mientras otro recoge el objeto encontrado

Colocar un objeto para que la sombra resultante indique una pista

Emplear la decoración para esconder pistas dentro de cuadros, mobiliario, etc.

Darse cuenta de una secuencia numérica identificando objetos de diferentes colores ubicados en la habitación

Esconder un mensaje leyendo el texto de forma particular (por ejemplo, las primeras letras de cada línea o mediante una plantilla perforada)

Se comparan determinados símbolos utilizando una clave e identificando con qué números o letras se corresponden

Encontrar por un lado un documento con unas coordenadas alfanuméricas y, por otro lado, un cuadrante con números en las filas y letras en las columnas cuyas celdas contienen alguna información. Combinando los dos elementos se define cuáles son las casillas que contienen información relevante

Usar una tarjeta de crédito para abrir una puerta sin pomo

Varios fragmentos de papel han de ser leídos en un determinado orden

Extraer un libro de una estantería para abrir un pasadizo secreto

Establecer una secuencia de forma que el siguiente objeto sea el que contiene la información necesaria para resolver un puzle

En un papel junto a un frutero se lee "Oro parece, plata no es". En el frutero hay varias frutas y dentro del plátano un código

Dar mensajes mediante código Morse o secuencias de sonidos que ayuden a resolver otro puzle

Usar imágenes o mensajes que solo se descifran reflejadas en un espejo

Llenar un recipiente con agua usando una pistola de agua para coger una pieza de corcho con una pista

Puzles en el que el reto es simplemente llegar a la respuesta correcta

La combinación de un candado es la fecha de nacimiento de un personaje que viene explícito en una biografía

Emplear cuerdas o cadenas para dificultar el acceso a determinados elementos o limitar la capacidad de los jugadores

Solucionar un crucigrama donde se obtiene una pista para el siguiente puzle

Utilizar herramientas para coger objetos inaccesibles (pinchas, palos, cuerdas con imanes) o espejos para leer algo que está fuera de la línea de visión

Utilizar un coche dirigido por control remoto para acercar un objeto inaccesible

Identificar un objeto dentro de un recipiente sellado empleando el tacto

Emplear el sentido del tacto para identificar objetos o manipular mecanismos

Construir una determinada figura usando piezas de un tangram

Algún actor interactúa con los jugadores proporcionando pistas 
juegos adquieren su mayor potencialidad didáctica. Lo importante es que el alumnado haya aprendido y disfrutado de la experiencia en sí, más que si ha logrado resolver el reto o no. Este tipo de actividades generan un alto grado de estrés, por lo que se recomienda un tiempo de distensión para poder compartir lo vivido por los alumnos y recoger algunas impre- siones sobre lo que ha sucedido, lo que han aprendido y que sean conscientes de todo lo que implica este tipo de actividad. Un paso más allá sería implicarles en el diseño de alguna mejora o alternativa para que lo realicen otros compañeros de otros cursos.

A modo de cierre, comentaremos una experiencia llevada a cabo en el aula:

La última sesión a la que se tuvieron que enfrentar los aspirantes del Ministerio del Tiempo fue un juego de escape. Su entrenamiento había comenzado al principio de la tercera y última evaluación. Un proyecto de gamificación realizado en el Colegio Luis Amigó, en Mutilva (Navarra), para abordar la parte más árida del temario de la asignatura de Religión Católica de $3 .^{\circ}$ de la ESO. Es decir, la Historia de la Iglesia, desde las primeras comunidades hasta el Gran Cisma de Occidente. Más de 13 siglos.

La actividad comienza con el aula precintada y con la indicación de que los alumnos deben ir a otra. Allí nos espera el ministro, protegido con un buzo y una mascarilla. Nos explica la misión con un video: el papa está en peligro. Jerusalén 1964. Un grupo terrorista ha preparado un ataque biológico contra el papa Pablo VI y el patriarca Atenágoras, en un encuentro que iba a poner fin a un distanciamiento de 1000 años entre la Iglesia católica y la Iglesia ortodoxa.

Equipados con mascarillas, regresamos al aula, donde el material del juego está repartido por las mesas. Son los mismos grupos que han trabajado en las misiones anteriores. Un temporizador se proyecta en la pizarra. Es el tiempo que tenemos antes de que explote la bomba con el patógeno. Disponen de la ayuda del libro. Candados, sobres, acertijos, mensajes ocultos y puzles. Con ellos van adentrándose en los contenidos del tema: el Cisma de Oriente. Su año, sus protagonistas y el porqué, además de sus consecuencias hasta el día de hoy y otros cismas posteriores.

El otro contenido: el del trabajo en grupo y el juego cooperativo, puesto que la bomba se desactivaba con los códigos adivinados por toda el aula. La concentración y la motivación de los grupos era máxima. Nos sobró tiempo, y mucho. Eso lo dice todo. Lo empleamos en repasar y afianzar lo aprendido, y en recoger. También en celebrar que habían logrado ser admitidos como agentes del Ministerio del Tiempo.

\section{DARA SABER MÂS}

Junta de Extremadura. (2018). Manual de Diseño de un Juego de Escape. Mérida: Instituto de la Juventud de Extremadura.

Negre I Walczak, C. (2017, 10 de diciembre). Guía definitiva para crear un \#breakout o \#escaperoom educativo. Applejux.org: analogue multimedia. Recuperado de https://www.applejux.org/2017/12/laguia-definitiva-para-crear-tu-breakoutedu-o-escaperoom/ [Disponible en castellano, catalán e inglés].

Quilez, J. (2020, 1 de marzo). Cómo crear paso a paso un EscapeRoom (tutorial) [presentación de diapositivas]. Slideshare. Recuperado de http://www.javiquil.com/archivos/5491

En la siguiente dirección se pueden encontrar diversas claves para generar códigos http://bestcodes.weebly.com y en este otro enlace se pueden crear fácilmente crucigramas para las pistas: https:// worksheets.theteacherscorner.net/make-your-own/crossword/ lang-es/

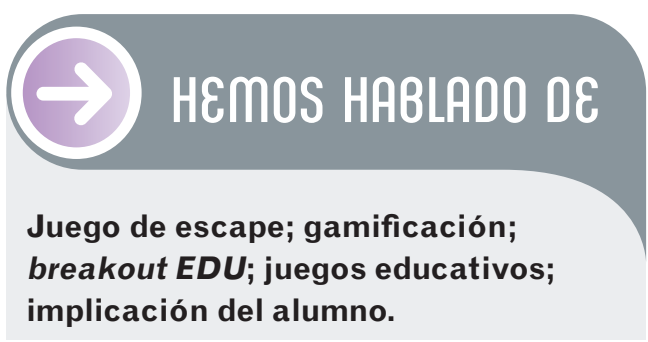

Este artículo fue solicitado por PADRES Y MAESTROS en marzo de 2020, revisado y aceptado en junio de 2020. 